\title{
\begin{tabular}{l|l} 
Mibraries & DSpace@MIT
\end{tabular}
}

\author{
MIT Open Access Articles
}

\section{An Offline-Online Riemann Solver for One- Dimensional Systems of Conservation Laws}

The MIT Faculty has made this article openly available. Please share how this access benefits you. Your story matters.

Citation: Taddei, Tommaso, Alfio Quarteroni, and Sandro Salsa. “An Offline-Online Riemann Solver for One-Dimensional Systems of Conservation Laws." Vietnam Journal of Mathematics 44, no. 4 (June 11, 2016): 873-891.

As Published: http://dx.doi.org/10.1007/s10013-016-0212-0

Publisher: Springer Singapore

Persistent URL: http://hdl.handle.net/1721.1/105761

Version: Author's final manuscript: final author's manuscript post peer review, without publisher's formatting or copy editing

Terms of Use: Article is made available in accordance with the publisher's policy and may be subject to US copyright law. Please refer to the publisher's site for terms of use. 


\title{
An Offline-Online Riemann Solver for One-Dimensional Systems of Conservation Laws
}

\author{
Tommaso Taddei ${ }^{1}$ - Alfio Quarteroni ${ }^{2,3}$. \\ Sandro Salsa ${ }^{4}$
}

Received: 9 January 2015 / Accepted: 23 February 2016

\begin{abstract}
In this paper, we present an exact Riemann solver for one-dimensional systems of conservation laws. The method is based on an offline-online computational decomposition. During the offline stage, we generate an accurate surrogate model for the solution to the Riemann problem for arbitrary left and right states. Then, during the online stage, we employ the surrogate model to generate accurate initial conditions for an iterative Newton solver. We present a mathematical analysis of the Riemann problem to justify the proposed approach. Finally, we illustrate its effectiveness by means of two numerical examples.
\end{abstract}

Keywords Nonlinear conservation laws · Model reduction · Riemann solvers

Mathematics Subject Classification $65 \mathrm{M} 08 \cdot 65 \mathrm{M} 25 \cdot 35 \mathrm{~L} 65 \cdot 35 \mathrm{~L} 67$

Tommaso Taddei

E-mail: ttaddei@mit.edu

Alfio Quarteroni

E-mail: alfio.quarteroni@epfl.ch

E-mail: alfio.quarteroni@polimi.it

Sandro Salsa

E-mail: sandro.salsa@polimi.it

1 Mechanical Engineering Department, Massachusetts Institute of Technology, 77 Mass Avenue, Cambridge, MA 02139, USA

2 Modelling and Scientific Computing, Institute of Analysis and Scientific Computing, École Polytechnique Féderale de Lausanne, Station 8, EPFL, CH-1015 Lausanne, Switzerland

3 MOX-Modellistica e Calcolo Scientifico, Dipartimento di Matematica "F. Brioschi" Politecnico di Milano, via Bonardi 9, 20133 Milano, Italy

4 Dipartimento di Matematica "F. Brioschi", Politecnico di Milano, via Bonardi 9, 20133 Milano, Italy 


\section{Introduction and Motivations}

A wide class of finite volume methods for one-dimensional systems of conservation laws $([4,6,17])$ requires the approximation of the solution to Riemann problems at cell interfaces between the neighboring cell averages. For Computational Fluid Dynamics (CFD) applications, the Riemann problem is solved an extremely large number of times, representing therefore the single most burdensome task in the numerical method ([16]).

Given the flux $\mathbf{F}: \mathbb{R}^{m} \mapsto \mathbb{R}^{m}$ and the initial left and right states $\mathbf{u}_{l}, \mathbf{u}_{r} \in \mathbb{R}^{m}$, the Riemann problem is defined as

$$
\mathbf{u}_{t}+\mathbf{F}(\mathbf{u})_{x}=0, \quad(x, t) \in \mathbb{R} \times(0, \infty),
$$

with initial condition

$$
\mathbf{u}(x, 0)=\mathbf{u}_{l} \quad \text { for } x<0, \quad \mathbf{u}(x, 0)=\mathbf{u}_{r} \quad \text { for } x \geq 0 .
$$

In this work, we consider $\mathbf{F} \in C^{2}\left(\mathbb{R}^{m} ; \mathbb{R}^{m}\right)$ for some integer $m \geq 2$. We also assume that the flux $\mathbf{F}$ is strictly hyperbolic, i.e., the Jacobian matrix $D \mathbf{F}$ is diagonizable with distinct real eigenvalues. Under suitable hypotheses, we show that the solution to (1a)-(1b) is uniquely determined by the solution $\widehat{\mathbf{u}}=\widehat{\mathbf{u}}\left(\mathbf{u}_{l}, \mathbf{u}_{r}\right) \in \mathbb{R}^{m^{2}}$ to the nonlinear system of algebraic equations

$$
\mathcal{L}\left(\widehat{\mathbf{u}} ; \mathbf{u}_{l}, \mathbf{u}_{r}\right)=\mathbf{0}, \quad \mathcal{L}: \mathbb{R}^{m^{2}} \times \mathbb{R}^{m} \times \mathbb{R}^{m} \mapsto \mathbb{R}^{m^{2}}
$$

For several differential problems, including the two systems considered in this paper, a priori considerations lead to a reduction of the dimensionality of the nonlinear algebraic system $\mathcal{L}$. More precisely, there exists a smooth bijective map between the solution $\widehat{\mathbf{u}}$ to $(2)$ and the solution $\widehat{\mathbf{u}}^{\text {red }}$ to the reduced system of nonlinear equations

$$
\mathcal{L}^{\text {red }}\left(\widehat{\mathbf{u}}^{\text {red }} ; \mathbf{u}_{l}, \mathbf{u}_{r}\right)=\mathbf{0}, \quad \mathcal{L}^{\text {red }}: \mathbb{R}^{p} \times \mathbb{R}^{m} \times \mathbb{R}^{m} \mapsto \mathbb{R}^{p},
$$

where $p<m^{2}$.

During the last fifty or sixty years, much effort has been devoted to develop efficient Riemann solvers either exact or approximate. Exact Riemann solvers are based on the solution of the system of algebraic nonlinear equations (3) via an iterative procedure. For the Euler equations, several different exact Riemann solvers have been proposed in the literature; we refer to [16, Chapter 4] and to [7] for a thorough discussion and numerical comparisons. Approximate Riemann solvers estimate the solution to the Riemann problem preserving some key properties of the physical solution. After the seminal work of Roe [14] for the Euler's equation, several approximate Riemann solvers have been proposed for a wide class of systems of conservation laws; we refer to [16] and to the references therein for further details. Exact Riemann solvers heavily rely on the accuracy of the initial guess of the solution to the system of nonlinear equations. On the other hand, approximate Riemann solvers are less accurate and possibly hard to derive.

In this work, we propose a general exact Riemann solver for one-dimensional systems of conservation laws. The strategy is based on the decomposition of the computational work into two different phases. In the first phase, called offline stage, we build an approximate surrogate model for the solution $\widehat{\mathbf{u}}^{\text {red }}$ to (3) for each choice 
of $\left(\mathbf{u}_{l}, \mathbf{u}_{r}\right)$ in $\mathcal{D}, \widehat{\mathbf{u}}^{\text {surrogate }}: \mathcal{D} \rightarrow \mathbb{R}^{m^{2}}$. Here, $\mathcal{D} \subset \mathbb{R}^{2 m}$ is a suitable compact set. The surrogate model $\widehat{\mathbf{u}}^{\text {surrogate }}$ is built by solving a regression problem based on the solution to several Riemann problems with left and right states $\left(\mathbf{u}_{l}, \mathbf{u}_{r}\right) \in \mathcal{D}$. In the second phase, called online stage, we solve multiple Riemann problems by using the surrogate model $\widehat{\mathbf{u}}^{\text {surrogate }}$ to compute the initial guess for the iterative solver. The online stage corresponds to the application of a finite volume scheme to the one-dimensional system of conservation laws (1a) with general initial condition.

We provide a rigorous mathematical justification of the proposed approach. We rigorously prove that, for the class of problems considered in this work, the solution $\widehat{\mathbf{u}}$ to $(2)$ depends smoothly on $\left(\mathbf{u}_{l}, \mathbf{u}_{r}\right)$. Since, by assumption, there exists a smooth bijective map between $\widehat{\mathbf{u}}$ in $(2)$ and $\widehat{\mathbf{u}}^{\text {red }}$ in $(3)$, this implies that $\widehat{\mathbf{u}}^{\text {red }}$ can be approximated through a smooth expansion in $\left(\mathbf{u}_{l}, \mathbf{u}_{r}\right)$.

We illustrate our method through two practical examples: the p-system and the Euler equations for ideal gases. For these two problems, we compare the initial guesses obtained using our offline/online method with the a priori initial guesses employed in state-of-the-art exact Riemann solvers, and we quantitatively discuss the influence of the initial guess on the performance of the Newton solver.

Offline/online computational procedures have been proposed and extensively analyzed in the context of Model Order Reduction for parametric partial differential equations (PDEs) $([2,9,13])$. In particular, our idea of developing an offlineonline computational strategy to provide an accurate initial guess for nonlinear Newton-like solvers has already been exploited in [3]. However, to our knowledge, this paper represents the first attempt to exploit this technique in the development of Riemann solvers for systems of conservation laws. Furthermore, unlike in [3], we do not directly apply the offline/online strategy to the solution to the PDE.

This paper is organized as follows. In Section 2, we introduce our method and we present the computational procedure. Then, in Section 3, we provide the theoretical justification of the proposed technique. In particular, we present an explicit expression for the system $\mathcal{L}$ in (2) and we show that $\mathbf{u}$ is differentiable in $\left(\mathbf{u}_{l}, \mathbf{u}_{r}\right)$. Finally, in Sections 4 and 5, we apply our method to the p-system and the Euler equations for ideal gases.

\section{Methodology}

In this section, we present the offline-online Riemann solver. First, in Section 2.1, we briefly review the key ideas of exact Riemann solvers for one-dimensional systems of conservation laws. Then, in Section 2.2, we detail the computational procedure. In order to simplify the notation, in what follows, we refer to the $2 \mathrm{~m}$ dimensional vector consisting of the left and right states of the Riemann problem $\left(\mathbf{u}_{l}, \mathbf{u}_{r}\right)$ as to $\boldsymbol{\mu}$.

\subsection{Exact Riemann Solvers for One-dimensional Systems of Conservation Laws}

As shown in Section 3 and recalled in the introduction, exact Riemann solvers exploit the fact that the solution to the Riemann problem (1a)-(1b) is uniquely determined by the solution $\widehat{\mathbf{u}}$ to the nonlinear system of algebraic equations (2). For several differential problems, we can simplify the nonlinear system (2) to obtain the 
reduced system (3). The latter system is then solved using an iterative nonlinear solver of the form

$$
\left[\widehat{\mathbf{u}}^{\text {red }}(\boldsymbol{\mu})\right]=\text { nonlinear-solver }\left(\mathcal{L}^{\text {red }}, \partial_{\widehat{\mathbf{u}}^{\text {red }}} \mathcal{L}^{\text {red }}, \widehat{\mathbf{u}}^{\text {guess }}, \boldsymbol{\mu}\right),
$$

where $\boldsymbol{\mu}=\left(\mathbf{u}_{l}, \mathbf{u}_{r}\right), \partial_{\widehat{\mathbf{u}}^{\text {red }}} \mathcal{L}^{\text {red }}$ denotes the partial derivatives of $\mathcal{L}^{\text {red }}$ with respect to the first argument, and $\widehat{\mathbf{u}}^{\text {guess }}$ sets the initial condition of the iterative procedure.

Some comments are in order. First, the explicit general formula of system $\mathcal{L}$ for one-dimensional systems of conservation laws is given in Section 3.1. On the other hand, in Sections 4 and 5, we derive $\mathcal{L}^{\text {red }}$ for the two problems considered in this work. Second, the performance of the iterative solver (4) strongly depends on the accuracy of the initial guess $\widehat{\mathbf{u}}^{\text {guess }}$. It is well known, indeed, ([12]) that, without a sufficiently accurate initial estimate, it is necessary to combine a high order and locally convergent method (e.g., Newton method) with a more robust root finding method (e.g., bisection or secant methods). The need for the latter classes of schemes significantly deteriorates the performance of the exact Riemann solver.

\subsection{An Offline-Online Strategy for the Resolution of the Riemann Problem}

\subsubsection{Offline-Online Strategy}

We first motivate the strategy proposed in this paper. As shown in Section 3.2, the solution $\widehat{\mathbf{u}}=\widehat{\mathbf{u}}(\boldsymbol{\mu})$ to (1a)-(1b) is differentiable in both arguments. As explained in the introduction, this implies that $\widehat{\mathbf{u}}^{\text {red }}$ can be approximated through a smooth surrogate expansion $\widehat{\mathbf{u}}^{\text {surrogate }}$ in $\boldsymbol{\mu}$.

Motivated by the above considerations, we can now propose our offline-online computational strategy. In the offline stage, we first solve the Riemann problem (1a)-(1b) for a certain number of states $\Xi_{N}:=\left\{\boldsymbol{\mu}_{i}=\left(\mathbf{u}_{l}^{i}, \mathbf{u}_{r}^{i}\right)\right\}_{i=1}^{N} \subset \mathcal{D} \subset \mathbb{R}^{2 m}$, where $\mathcal{D}$ is a compact parameter set. Then, we use the dataset $\left\{\left(\boldsymbol{\mu}_{i}, \widehat{\mathbf{u}}^{\text {red }}\left(\boldsymbol{\mu}_{i}\right)\right\}_{i=1}^{N}\right.$ to generate the surrogate model $\widehat{\mathbf{u}}^{\text {surrogate }}: \mathcal{D} \rightarrow \mathbb{R}^{p}$. The latter is a regression problem where $\boldsymbol{\mu}$ represents the vector of independent (or explanatory) variables and $\widehat{\mathbf{u}}^{\text {red }}$ is the vector of dependent (or response) variables. In Section 2.2.2, we present the regression procedure employed in this work to solve this problem. In the online stage, which corresponds to the application of a finite volume scheme to the one-dimensional system of conservation laws (1a) with general initial conditions, we can then use the surrogate model to generate accurate initial guesses for the iterative solver (4).

\subsubsection{Construction of the Surrogate Model}

In view of the presentation of the regression procedure, we first introduce some notation. First, we introduce the dataset $\mathcal{P}_{N}=\left\{\left(\boldsymbol{\mu}_{i}, \widehat{\mathbf{u}}_{i}=\widehat{\mathbf{u}}\left(\boldsymbol{\mu}_{i}\right)\right)\right\}_{i=1}^{N}$. Furthermore, we define the training set $\mathcal{P}_{N_{\text {train }}^{\text {train }}}^{\text {trat }}=\left\{\left(\boldsymbol{\mu}_{i}^{\text {train }}, \widehat{\mathbf{u}}_{i}^{\text {train }}\right\}_{i=1}^{N_{\text {train }} \text {, the validation set }}\right.$ $\mathcal{P}_{N_{\text {val }}}^{\text {val }}=\left\{\left(\boldsymbol{\mu}_{i}^{\text {val }}, \widehat{\mathbf{u}}_{i}^{\text {val }}\right\}_{i=1}^{N_{\text {val }}}\right.$, and the test set $\mathcal{P}_{N_{\text {test }}}^{\text {test }}=\left\{\left(\boldsymbol{\mu}_{i}^{\text {test }}, \widehat{\mathbf{u}}_{i}^{\text {test }}\right\}_{i=1}^{N_{\text {test }}}\right.$ such that $\mathcal{P}_{N_{\text {train }}}^{\text {train }}, \mathcal{P}_{N_{\text {val }}}^{\text {val }}, \mathcal{P}_{N_{\text {test }}}^{\text {test }}$ are a partition of $\mathcal{P}_{N}$. 
In this work, we employ Kernel Ridge regression based on Gaussian kernels (see. e.g., [11, Chapter 14.4.3]). The procedure seeks for a surrogate model of the form

$$
\widehat{\mathbf{u}}^{\text {surrogate }}(\boldsymbol{\mu})=\sum_{i=1}^{N_{\text {train }}}\left(\sum_{k=1}^{p} \alpha_{i, k} \mathbf{e}_{k}\right) K_{\lambda}\left(\boldsymbol{\mu}, \boldsymbol{\mu}_{i}^{\text {train }}\right),
$$

where $\mathbf{e}_{k}$ are the vectors of the canonical basis of $\mathbb{R}^{p}, K_{\lambda}\left(\boldsymbol{\mu}, \boldsymbol{\mu}^{\prime}\right)=\exp (-\lambda \| \boldsymbol{\mu}-$ $\left.\boldsymbol{\mu}^{\prime} \|_{2}^{2}\right)$ is the Gaussian kernel, and $\left\{\alpha_{i, k}\right\}_{i, k}$ is a matrix of coefficients to be determined. Given $\xi>0$, in Kernel Ridge regression, the coefficients $\left\{\alpha_{i, k}\right\}_{i, k}$ are computed by solving the $p$ linear systems:

$$
(\mathbb{K}+\xi \mathbb{I}) \boldsymbol{\alpha}^{k}=\mathbf{b}^{k}, \quad k=1, \ldots, p,
$$

where $\mathbb{K}_{i, i^{\prime}}=K_{\lambda}\left(\boldsymbol{\mu}_{i}^{\text {train }}, \boldsymbol{\mu}_{i^{\prime}}^{\text {train }}\right),\left(\boldsymbol{\alpha}^{k}\right)_{i}=\alpha_{i, k}$ and $\left(\mathbf{b}^{k}\right)_{i}=\left(\widehat{\mathbf{u}}^{\text {red }}\left(\boldsymbol{\mu}_{i}\right)\right)_{k}$ for $i, i^{\prime}=$ $1, \ldots, N_{\text {train }}$.

We now discuss how to tune the parameters of the regression procedure and how to assess its performance. First, we observe that the regression procedure depends on two parameters: the kernel parameter $\lambda$ and the penalization weight $\xi$. These parameters can be chosen by minimizing the mean square error associated with the surrogate model $\widehat{\mathbf{u}}^{\text {surrogate }}$ over the validation set

$$
M S E^{\mathrm{val}}:=\frac{1}{N_{\text {val }}} \sum_{i=1}^{N_{\mathrm{val}}}\left\|\widehat{\mathbf{u}}^{\mathrm{red}}\left(\boldsymbol{\mu}_{i}^{\mathrm{val}}\right)-\widehat{\mathbf{u}}^{\text {surrogate }}\left(\boldsymbol{\mu}_{i}^{\mathrm{val}}\right)\right\|_{2}^{2} .
$$

On the other hand, in order to assess the accuracy of our estimate, we compute the mean square error over the test set

$$
M S E^{\text {test }}:=\frac{1}{N_{\text {test }}} \sum_{i=1}^{N_{\text {test }}}\left\|\widehat{\mathbf{u}}^{\text {red }}\left(\boldsymbol{\mu}_{i}^{\text {test }}\right)-\widehat{\mathbf{u}}^{\text {surrogate }}\left(\boldsymbol{\mu}_{i}^{\text {test }}\right)\right\|_{2}^{2} .
$$

We recall (see, e.g., [15]) that if the test points $\boldsymbol{\mu}_{i}^{\text {test }}$ are drawn from a uniform distribution over $\mathcal{D}$, then $M S E^{\text {test }}$ is a Monte Carlo approximation of the integral

$$
M S E:=\int_{\mathcal{D}}\left\|\widehat{\mathbf{u}}^{\text {red }}(\boldsymbol{\mu})-\widehat{\mathbf{u}}^{\text {surrogate }}(\boldsymbol{\mu})\right\|_{2}^{2} d \boldsymbol{\mu} .
$$

Remark 1 (Adaptive choice of $N$ ) The evaluation of the mean square error over the test set can be exploited to adaptively choose the value of $N$ following the idea proposed in [8] in the framework of reduced basis method. The key idea is to specify a priori a threshold $t o l>0$ and then iteratively enlarge the training set $\Xi_{N}$ until $M S E^{\text {test }}<$ tol.

\subsubsection{Computational Procedure}

In Algorithm 1, we summarize the procedure. In order to simplify the presentation of the computational procedure, we refer to the application of the regression procedure described in Section 2.2.2 as to

$$
\left[\widehat{\mathbf{u}}^{\text {surrogate }}\right]=\text { Kernel-Ridge-Regression }\left(\mathcal{P}_{N_{\text {train }}}^{\text {train }}, \mathcal{P}_{N_{\text {val }}^{\text {val }}}^{\text {val }}\right)
$$




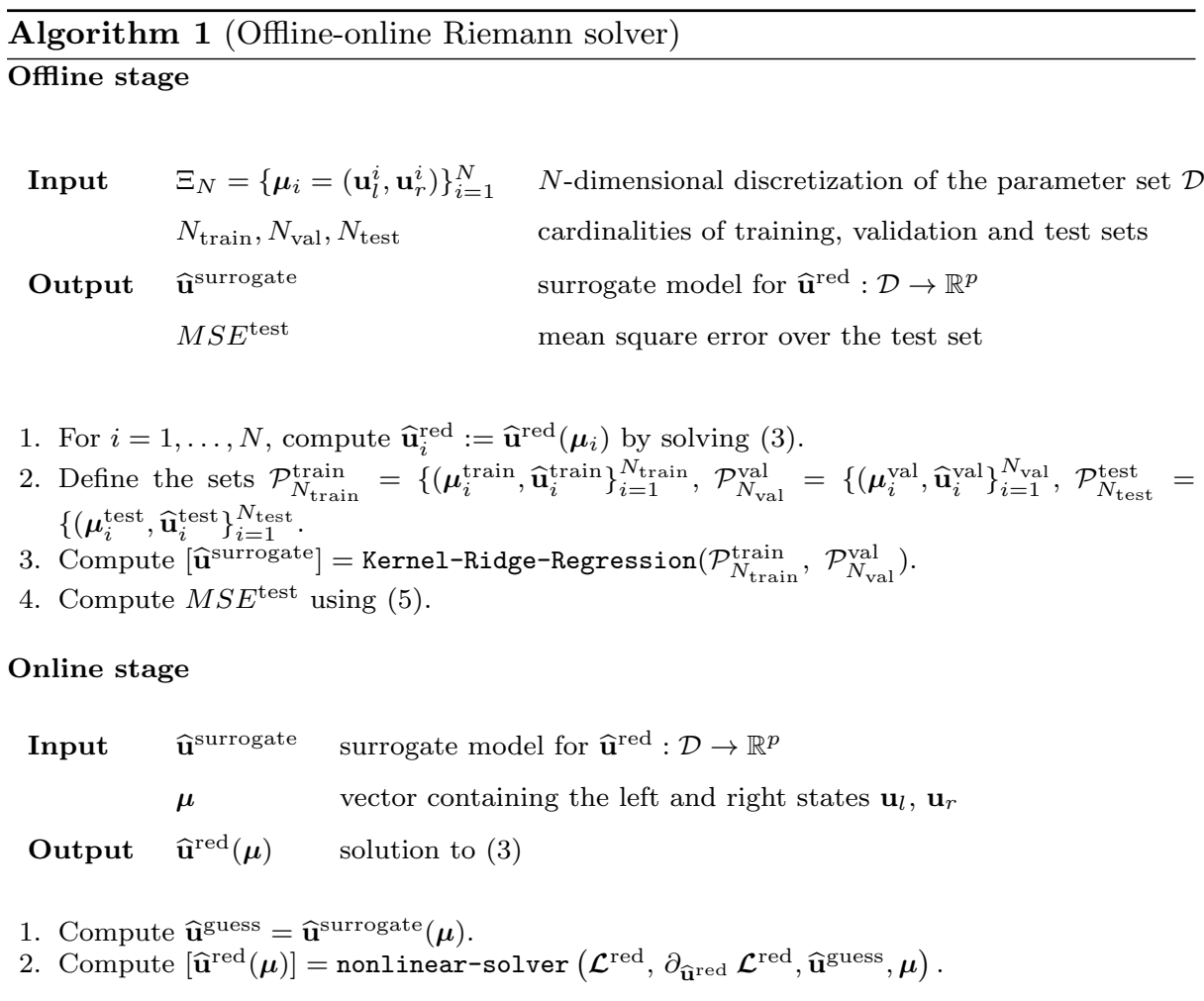

\section{Analysis of the Riemann Problem}

In this section, we provide the mathematical foundations for the computational algorithm developed in Section 2. First, in Section 3.1, we show that, if $\mathbf{u}_{l}, \mathbf{u}_{r}$ are sufficiently close to each other, then the solution to (1a)-(1b) exists, is unique and is completely determined by the solution to an algebraic system of equations of the form (2). Then, in Section 3.2, we prove that the solution $\widehat{\mathbf{u}}$ to (2) depends smoothly on the left and right states. Our analysis is largely inspired by $[5,10]$.

\subsection{Solution to the Riemann Problem}

We first introduce the characteristics of equation (1a). Towards this end, for all $\mathbf{z} \in \mathbb{R}^{m}$ and $k=1, \ldots, m$, we introduce the triplets $\left(\lambda_{k}, \mathbf{r}_{k}, \mathbf{l}_{k}\right)$ such that

$$
D \mathbf{F}(\mathbf{z}) \mathbf{r}_{k}(\mathbf{z})=\lambda_{k}(\mathbf{z}) \mathbf{r}_{k}(\mathbf{z}), \quad \lambda_{1}(\mathbf{z})<\cdots<\lambda_{m}(\mathbf{z}) .
$$

We suppose that, for each $k=1, \ldots, m$, the eigenpair $\left(\lambda_{k}, \mathbf{r}_{k}\right)$ satisfies one of the following conditions:

$$
\begin{aligned}
& \left\{\nabla \lambda_{k}(\mathbf{z}) \cdot \mathbf{r}_{k}(\mathbf{z}) \neq 0 \quad \forall \mathbf{z} \in \mathbb{R}^{m} \quad\right. \text { (genuinely nonlinear eigenpair), } \\
& \left\{\nabla \lambda_{k}(\mathbf{z}) \cdot \mathbf{r}_{k}(\mathbf{z})=0 \quad \forall \mathbf{z} \in \mathbb{R}^{m} \quad\right. \text { (linearly degenerate eigenpair). }
\end{aligned}
$$


Given $\mathbf{u}_{l} \in \mathbb{R}^{m}$, we define the $k$-th rarefaction curve $\mathbf{R}_{k}\left(\mathbf{u}_{l}\right)$ as the path in $\mathbb{R}^{m}$ of the solution to the $\mathrm{ODE} \mathbf{v}^{\prime}(s)=\mathbf{r}_{k}(\mathbf{v}(s))$ in a neighborhood of $s=0$, with initial condition $\mathbf{v}(0)=\mathbf{u}_{l}$ :

$$
\mathbf{R}_{k}\left(\mathbf{u}_{l}\right):=\left\{\mathbf{v}(s): \mathbf{v}^{\prime}(s)=\mathbf{r}_{k}(\mathbf{v}(s)), \mathbf{v}(0)=\mathbf{u}_{l} ; s \in(-\delta, \delta)\right\},
$$

where $\delta>0$. Furthermore, we define the shock set

$$
\mathbf{S}\left(\mathbf{u}_{l}\right)=\left\{\mathbf{z} \in \mathbb{R}^{m}: \mathbf{F}(\mathbf{z})-\mathbf{F}\left(\mathbf{u}_{l}\right)=\sigma\left(\mathbf{z}-\mathbf{u}_{l}\right) \text { for a constant } \sigma=\sigma\left(\mathbf{z}, \mathbf{u}_{l}\right)\right\} .
$$

It is possible to show (see [5, Theorem 2, Chapter 11.2.3]) that the shock set $\mathbf{S}\left(\mathbf{u}_{l}\right)$ consists of the union of $m$ differentiable curves $\mathbf{S}_{k}\left(\mathbf{u}_{l}\right), k=1, \ldots, m$, such that each curve $\mathbf{S}_{k}\left(\mathbf{u}_{l}\right)$ passes through $\mathbf{u}_{l}$, with tangent $\mathbf{r}_{k}\left(\mathbf{u}_{l}\right)$. It follows that the curves $\mathbf{R}_{k}\left(\mathbf{u}_{l}\right)$ and $\mathbf{S}_{k}\left(\mathbf{u}_{l}\right)$ agree at least to first order at $\mathbf{u}_{l}$. In the linearly degenerate case, these curves in fact coincide.

We now present the definition of entropic solution to the Riemann problem (1a)-(1b). First, we introduce the sets

$$
\left\{\begin{array}{l}
\mathbf{R}_{k}^{+}\left(\mathbf{u}_{l}\right)=\left\{\mathbf{z} \in \mathbf{R}_{k}\left(\mathbf{u}_{l}\right): \lambda_{k}(\mathbf{z})>\lambda_{k}\left(\mathbf{u}_{l}\right)\right\} \\
\mathbf{R}_{k}^{-}\left(\mathbf{u}_{l}\right)=\left\{\mathbf{z} \in \mathbf{R}_{k}\left(\mathbf{u}_{l}\right): \lambda_{k}(\mathbf{z})<\lambda_{k}\left(\mathbf{u}_{l}\right)\right\} \\
\mathbf{S}_{k}^{+}\left(\mathbf{u}_{l}\right)=\left\{\mathbf{z} \in \mathbf{S}_{k}\left(\mathbf{u}_{l}\right): \lambda_{k}\left(\mathbf{u}_{l}\right)<\sigma\left(\mathbf{z}, \mathbf{u}_{l}\right)<\lambda_{k}(\mathbf{z})\right\} \\
\mathbf{S}_{k}^{-}\left(\mathbf{u}_{l}\right)=\left\{\mathbf{z} \in \mathbf{S}_{k}\left(\mathbf{u}_{l}\right): \lambda_{k}(\mathbf{z})<\sigma\left(\mathbf{z}, \mathbf{u}_{l}\right)<\lambda_{k}\left(\mathbf{u}_{l}\right)\right\}
\end{array}\right.
$$

and the curves $\mathbf{T}_{k}\left(\mathbf{u}_{l}\right)$ such that

$$
\mathbf{T}_{k}\left(\mathbf{u}_{l}\right):=\mathbf{R}_{k}^{+}\left(\mathbf{u}_{l}\right) \cup\left\{\mathbf{u}_{l}\right\} \cup \mathbf{S}_{k}^{-}\left(\mathbf{u}_{l}\right)
$$

for $k=1, \ldots, m$. If $\left(\lambda_{k}, \mathbf{r}_{k}\right)$ is genuinely nonlinear, recalling that the curves $\mathbf{R}_{k}\left(\mathbf{u}_{l}\right)$ and $\mathbf{S}_{k}\left(\mathbf{u}_{l}\right)$ agree at least to first order at $\mathbf{u}_{l}$, we have that the curve $\mathbf{T}_{k}\left(\mathbf{u}_{l}\right)$ admits a $C^{1}$ (and injective) parametrization $\epsilon \mapsto \mathbf{\Upsilon}_{k}\left(\epsilon, \mathbf{u}_{l}\right)$ such that $\left\{\mathbf{\Upsilon}_{k}\left(\epsilon, \mathbf{u}_{l}\right)\right.$ : $\epsilon>0\}=\mathbf{R}_{k}^{+}\left(\mathbf{u}_{l}\right)$ and $\left\{\boldsymbol{\Upsilon}_{k}\left(\epsilon, \mathbf{u}_{l}\right): \epsilon<0\right\}=\mathbf{S}_{k}^{-}\left(\mathbf{u}_{l}\right)$. On the other hand, if $\left(\lambda_{k}, \mathbf{r}_{k}\right)$ is linearly degenerate, $\mathbf{T}_{k}\left(\mathbf{u}_{l}\right):=\mathbf{R}_{k}\left(\mathbf{u}_{l}\right)=\mathbf{S}_{k}\left(\mathbf{u}_{l}\right)$ and it is still possible to parameterize the curve through a regular injective function $\epsilon \mapsto \mathbf{\Upsilon}_{k}\left(\epsilon, \mathbf{u}_{l}\right)$. We observe that each curve $\mathbf{T}_{k}\left(\mathbf{u}_{l}\right)$ glues together the physically relevant parts of the $k$-th rarefaction and $k$-th shock curve. We have now the elements to define the "physical" (entropic) solution to the Riemann problem (1a)-(1b) as in [5].

Definition $1 \mathbf{u} \in L^{\infty}\left(\mathbb{R} \times(0, \infty) ; \mathbb{R}^{m}\right)$ is said to be an integral solution to (1a)(1b) if it satisfies

$$
\int_{0}^{\infty} \int_{\mathbb{R}} \mathbf{u} \cdot \mathbf{v}_{t}+\mathbf{F}(\mathbf{u}) \cdot \mathbf{v}_{x} d x d t+\left.\int_{-\infty}^{0} \mathbf{u}_{l} \cdot \mathbf{v}\right|_{t=0} d x+\left.\int_{0}^{\infty} \mathbf{u}_{r} \cdot \mathbf{v}\right|_{t=0} d x=0
$$

for all $\mathbf{v} \in C_{0}^{1}\left(\mathbb{R}^{2} ; \mathbb{R}^{m}\right)$. Furthermore ${ }^{1}, \mathbf{u}$ is said to be admissible (or entropic) if for all $(x, t) \in \mathbb{R} \times(0, \infty)$ the left and right limits $\mathbf{u}^{ \pm}(x, t):=\lim _{y \rightarrow x^{ \pm}} \mathbf{u}(y, t)$ do exist and, if $\mathbf{u}^{+}(x, t) \neq \mathbf{u}^{-}(x, t)$, then $\mathbf{u}^{+}(x, t) \in \mathbf{S}_{k}^{-}\left(\mathbf{u}^{-}(x, t)\right)$ for some $k \in\{1, \ldots, m\}$.

On the ground of the previous properties and definitions, we can introduce three special types of solutions to problem (1a)-(1b), (see [5]).

\footnotetext{
1 This entropy condition is well suited for the solution to the Riemann problem. We refer to [5, Chapter 11.4] for more general entropy criteria.
} 
Lemma 1 Let $\left(\lambda_{k}, \mathbf{r}_{k}\right)$ be linearly degenerate and let $\mathbf{u}_{r} \in \mathbf{S}_{k}\left(\mathbf{u}_{l}\right)$ for some $k \in$ $\{1, \ldots, m\}$. Then

$$
\mathbf{u}(x, t)=\left\{\begin{array}{ll}
\mathbf{u}_{l}, & x<\sigma t, \\
\mathbf{u}_{r}, & x>\sigma t,
\end{array} \quad \sigma=\lambda_{k}\left(\mathbf{u}_{l}\right)=\lambda_{k}\left(\mathbf{u}_{r}\right)\right.
$$

is called $k$-contact discontinuity and it represents an admissible integral solution to the Riemann problem (1a)-(1b).

Lemma 2 Let $\left(\lambda_{k}, \mathbf{r}_{k}\right)$ be genuinely nonlinear and let $\mathbf{u}_{r} \in \mathbf{S}_{k}^{-}\left(\mathbf{u}_{l}\right)$. Then

$$
\mathbf{u}(x, t)=\left\{\begin{array}{ll}
\mathbf{u}_{l}, & x<\sigma t \\
\mathbf{u}_{r}, & x>\sigma t,
\end{array} \quad \sigma=\sigma\left(\mathbf{u}_{l}, \mathbf{u}_{r}\right)\right.
$$

is called $k$-shock wave and is an admissible integral solution to the Riemann problem $(1 \mathrm{a})-(1 \mathrm{~b})$.

Lemma 3 Let $\left(\lambda_{k}, \mathbf{r}_{k}\right)$ be genuinely nonlinear and let $\mathbf{u}_{r} \in \mathbf{R}_{k}^{+}\left(\mathbf{u}_{l}\right)$. Then, there exists a continuous admissible integral solution $\mathbf{u}$ to the Riemann problem (1a)(1b), which is a $k$-simple wave constant along lines through the origin. More precisely, given $\Theta_{l}, \Theta_{r} \in \mathbb{R}, \Theta_{l}<\Theta_{r}$, the solution to (1a)-(1b) is

$$
\mathbf{u}(x, t)=\mathbf{v}\left(\Theta\left(\frac{x}{t}\right)\right)
$$

where the function $\mathbf{v}:\left[\Theta_{l}, \Theta_{r}\right] \rightarrow \mathbb{R}^{m}$ satisfies

$$
\mathbf{v}\left(\Theta_{l}\right)=\mathbf{u}_{l}, \quad \mathbf{v}\left(\Theta_{r}\right)=\mathbf{u}_{r}, \quad \mathbf{v}^{\prime}(s)=\mathbf{r}_{k}(\mathbf{v}(s)), \quad s \in\left(\Theta_{l}, \Theta_{r}\right),
$$

while the function $\Theta: \mathbb{R} \mapsto \mathbb{R}$ is defined as

$$
\Theta(s):= \begin{cases}\Theta_{l}, & s<F_{k}^{\prime}\left(\Theta_{l}\right), \\ G_{k}(s), & F_{k}^{\prime}\left(\Theta_{l}\right) \leq s<F_{k}^{\prime}\left(\Theta_{r}\right), \\ \Theta_{r}, & s \geq F_{k}^{\prime}\left(\Theta_{r}\right),\end{cases}
$$

with $F_{k}(s)=\int_{0}^{s} \lambda_{k}(\mathbf{v}(t)) d t$ and $G_{k}=\left(F_{k}^{\prime}\right)^{-1}$.

We refer to this solution as to $k$-th rarefaction wave.

Provided that the states $\mathbf{u}_{l}, \mathbf{u}_{r} \in \mathbb{R}^{m}$ are sufficiently close to each other, we now characterize the solution to (1a)-(1b) as a sequence of rarefaction waves, shock waves, and/or contact discontinuities. Towards this end, we first introduce the map

$$
\boldsymbol{\epsilon}=\left(\epsilon_{1}, \ldots, \epsilon_{m}\right) \mapsto \mathbf{\Upsilon}\left(\boldsymbol{\epsilon}, \mathbf{u}_{l}\right)
$$

such that

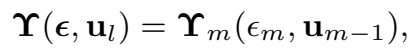

where $\mathbf{u}_{1}=\boldsymbol{\Upsilon}_{1}\left(\epsilon_{1}, \mathbf{u}_{l}\right), \mathbf{u}_{2}=\boldsymbol{\Upsilon}_{2}\left(\epsilon_{2}, \mathbf{u}_{1}\right), \ldots, \mathbf{u}_{m-1}=\boldsymbol{\Upsilon}_{m-1}\left(\epsilon_{m-1}, \mathbf{u}_{m-2}\right)$, and $\boldsymbol{\Upsilon}_{k}$ are the $C^{1}$-parametrizations of the curves $\mathbf{T}_{k}(\cdot)$ defined in (6). Moving from the definition of the map $\boldsymbol{\Upsilon}$, the next proposition provides an existence and uniqueness result for the solution to (1a)-(1b). We refer to [5, Theorem 4, Chapter 11.2.4] for the proof. 
Proposition 1 For each $k=1, \ldots, m$ assume that the pair $\left(\lambda_{k}, \mathbf{r}_{k}\right)$ is either genuinely nonlinear or else linearly degenerate. Suppose further the left state $\mathbf{u}_{l}$ is given.

Then, there exists a neighborhood $\mathcal{N}\left(\mathbf{u}_{l}\right)$ of the left state $\mathbf{u}_{l}$ such that if $\mathbf{u}_{r} \in$ $\mathcal{N}\left(\mathbf{u}_{l}\right)$, there exists a unique admissible (entropic) integral solution $\mathbf{u} \in L^{\infty}(\mathbb{R} \times$ $\left.(0, \infty) ; \mathbb{R}^{m}\right)$ to the Riemann problem, which is constant on lines through the origin.

Furthermore, the solution consists of the (possibly coincident) states $\mathbf{u}_{l}, \mathbf{u}_{1}, \ldots, \mathbf{u}_{m-1}, \mathbf{u}_{r}$, which are separated by contact discontinuities, shock waves or rarefaction waves, and there exists $\boldsymbol{\epsilon} \in \mathbb{R}^{m}$ such that $\mathbf{u}_{1}=\mathbf{\Upsilon}_{1}\left(\epsilon_{1}, \mathbf{u}_{l}\right), \ldots, \mathbf{u}_{r}=\mathbf{\Upsilon}_{m}\left(\epsilon_{m}, \mathbf{u}_{m-1}\right)$.

The next corollary contains the explicit expression of the function $\mathcal{L}$ anticipated in $(2)$.

Corollary 1 The solution to (1a)-(1b) is uniquely determined by the solution $\widehat{\mathbf{u}}=\left(\boldsymbol{\epsilon}^{\star}, \mathbf{u}_{1}, \ldots, \mathbf{u}_{m-1}\right) \in \mathbb{R}^{m^{2}}$ to the algebraic system of equations

$$
\mathcal{L}(\hat{\mathbf{u}} ; \boldsymbol{\mu})=\mathbf{0}, \quad \mathcal{L}(\hat{\mathbf{u}} ; \boldsymbol{\mu})=\left\{\begin{array}{l}
\mathbf{u}_{1}-\mathbf{\Upsilon}_{1}\left(\epsilon_{1}^{\star}, \mathbf{u}_{l}\right), \\
\mathbf{u}_{k}-\mathbf{\Upsilon}_{k}\left(\epsilon_{k}^{\star}, \mathbf{u}_{k-1}\right), \quad k=2, \ldots, m-1, \\
\mathbf{u}_{r}-\mathbf{\Upsilon}_{m}\left(\epsilon_{m}^{\star}, \mathbf{u}_{m-1}\right) .
\end{array}\right.
$$

Proof We observe that $\mathcal{L}(\hat{\mathbf{u}} ; \boldsymbol{\mu})=\mathbf{0}$ if and only if $\mathbf{u}_{r}=\boldsymbol{\Upsilon}\left(\boldsymbol{\epsilon}, \mathbf{u}_{l}\right)$. As a consequence, the system of algebraic equations (12) admits a unique solution $\widehat{\mathbf{u}} \in \mathbb{R}^{m^{2}}$. Furthermore, the solution $\widehat{\mathbf{u}} \in \mathbb{R}^{m^{2}}$ to (12) identifies the unique admissible solution to the Riemann problem (1a)-(1b).

We observe that the minimum information required to compute the solution to (1a)-(1b) consists of the states $\mathbf{u}_{1}, \ldots, \mathbf{u}_{m-1}$ and of the sign of the constants $\epsilon_{k}^{\star}$ such that the eigenpair $\left(\lambda_{k}, \mathbf{r}_{k}\right)$ is genuinely nonlinear. We have indeed that if the eigenpair $\left(\lambda_{k}, \mathbf{r}_{k}\right)$ is genuinely nonlinear the states $\mathbf{u}_{k-1}$ and $\mathbf{u}_{k}$ are pieced together by a $k$-th rarefaction wave if $\epsilon_{k}^{\star}>0$ and by a $k$-th shock if $\epsilon_{k}^{\star}<0$. Then, recalling Lemmas 1,2 , and 3 , we can build an explicit expression for the integral solution $\mathbf{u}(x, t)$ to $(7)$.

\subsection{Continuous Dependence of the Solution to Initial Conditions}

In this section, we show that the solution to (12) depends smoothly on the left and right states $\mathbf{u}_{l}$ and $\mathbf{u}_{r}$. Proposition 2 states the rigorous result.

Proposition $\mathbf{2}$ Assume the system (1a) is strictly hyperbolic and let $\mathbf{u}_{l}, \mathbf{u}_{r} \in \mathbb{R}^{m}$.

Then, if $\mathbf{u}_{l}, \mathbf{u}_{r}$ are sufficiently close to each other, there exists a neighborhood $\mathcal{N}(\boldsymbol{\mu}) \subset \mathbb{R}^{2 m}, \boldsymbol{\mu}=\left(\mathbf{u}_{l}, \mathbf{u}_{r}\right)$, such that the solution $\widehat{\mathbf{u}}$ to (12) is differentiable, that is $\widehat{\mathbf{u}} \in C^{1}\left(\mathcal{N}(\boldsymbol{\mu}) ; \mathbb{R}^{m^{2}}\right)$.

Before proving Proposition 2, we state an important corollary that follows from the above theorem.

Corollary 2 Let us assume that there exists a differentiable map $\mathcal{J}: \mathbb{R}^{m^{2}} \times$ $\mathbb{R}^{2 m} \mapsto \mathbb{R}^{p}$ such that $\widehat{\mathbf{u}}^{\text {red }}(\boldsymbol{\mu})=\mathcal{J}(\widehat{\mathbf{u}}(\boldsymbol{\mu}), \boldsymbol{\mu})$ in a neighborhood of $\boldsymbol{\mu}$. Then, under the hypotheses of Proposition 2, there exists a neighborhood $\mathcal{N}(\boldsymbol{\mu}) \subset \mathbb{R}^{2 m}$ such that the solution $\widehat{\mathbf{u}}^{\text {red }}$ to (3) is differentiable. 
In the remainder of this section, we present the proof of Proposition 2. First, we state the following lemma.

Lemma 4 (Dependence of eigenvalues and eigenvectors on parameters) Let $\mathbf{B} \in$ $C^{1}\left(\mathbb{R}^{\ell} ; \mathbb{R}^{m, m}\right), m, \ell \in \mathbb{N}$. For a given $\mathbf{z}_{0} \in \mathbb{R}^{\ell}$, let $\mathbf{B}\left(\mathbf{z}_{0}\right)$ be diagonizable with distinct eigenvalues $\lambda_{1}\left(\mathbf{z}_{0}\right)<\cdots<\lambda_{m}\left(\mathbf{z}_{0}\right)$.

Then, there exists a neighborhood $\mathcal{N}\left(\mathbf{z}_{0}\right)$ of $\mathbf{z}_{0}$ such that the eigenvalues $\lambda_{k}$ and the left and right eigenvectors $\mathbf{l}_{k}, \mathbf{r}_{k}$ are differentiable in $\mathcal{N}\left(\mathbf{z}_{0}\right), \lambda_{k} \in C^{1}\left(\mathcal{N}\left(\mathbf{z}_{0}\right) ; \mathbb{R}\right)$, $\mathbf{l}_{k}, \mathbf{r}_{k} \in C^{1}\left(\mathcal{N}\left(\mathbf{z}_{0}\right) ; \mathbb{R}^{\ell}\right)$ for each $k \in\{1, \ldots, m\}$ and for all $\mathbf{z} \in \mathcal{N}\left(\mathbf{z}_{0}\right)$. Furthermore, the left and right eigenvectors $\mathbf{l}_{k}, \mathbf{r}_{k}$ satisfy the normalization $\left\|\mathbf{r}_{k}(\mathbf{z})\right\|_{2},\left\|\mathbf{l}_{k}(\mathbf{z})\right\|_{2}=$ 1 for each $k \in\{1, \ldots, m\}$ and for all $\mathbf{z} \in \mathcal{N}\left(\mathbf{z}_{0}\right)$.

The proof of Lemma 4 is a straightforward application of the Implicit Function Theorem to the system of equations $\mathbf{\Phi}(\mathbf{r}, \lambda, \mathbf{z})=\left(\mathbf{B}(\mathbf{z}) \mathbf{r}-\lambda \mathbf{r},\|\mathbf{r}\|_{2}^{2}-1\right)=\mathbf{0}$. We refer to [5, Theorem 2, Chapter 11.1.2] for the details of the proof.

Thanks to Lemma 4, we can prove that the function $\left(\boldsymbol{\epsilon}, \mathbf{u}_{l}\right) \mapsto \mathbf{\Upsilon}\left(\boldsymbol{\epsilon}, \mathbf{u}_{l}\right)$ is smooth with respect to the second argument, $\mathbf{u}_{l}$. To show this, we prove that for each $k=1, \ldots, m$, the curves $\mathbf{T}_{k}\left(\mathbf{u}_{l}\right)=\mathbf{R}_{k}^{+}\left(\mathbf{u}_{l}\right) \cup\left\{\mathbf{u}_{l}\right\} \cup \mathbf{S}_{k}^{-}\left(\mathbf{u}_{l}\right)$ are of class $C^{1}$ with respect to $\mathbf{u}_{l}$. Recalling the definition of $\boldsymbol{\Upsilon}$ in (11), the latter implies that the map $\Upsilon$ is a regular function of $\mathbf{u}_{l}$. By definition, it is straightforward that the curve $\mathbf{R}_{k}\left(\mathbf{u}_{l}\right)$ and the corresponding parameterization depend smoothly on $\mathbf{u}_{l}$. On the other hand, the continuous dependence of the shock set is less evident and is addressed by the next lemma.

Lemma 5 Let us consider the strictly hyperbolic system (1a).

Then, for each $k=1, \ldots, m$ there exists $\boldsymbol{\Psi}_{k}: I \times \mathbb{R}^{m} \rightarrow \mathbb{R}^{m}$ such that for each $\mathbf{u}_{l} \in \mathbb{R}^{m} \boldsymbol{\Psi}_{k}\left(\cdot, \mathbf{u}_{l}\right): I \rightarrow \mathbb{R}^{m}$ is a parameterization of $\mathbf{S}_{k}\left(\mathbf{u}_{l}\right)$ and $\boldsymbol{\Psi}_{k}\left(0, \mathbf{u}_{l}\right)=$ $\mathbf{u}_{l}$. Furthermore, there exists a neighborhood $\mathcal{N}\left(0, \mathbf{u}_{l}\right)$ of $\left(0, \mathbf{u}_{l}\right)$ such that $\mathbf{\Psi}_{k} \in$ $C^{1}\left(\mathcal{N}\left(0, \mathbf{u}_{l}\right) ; \mathbb{R}^{m}\right), k=1, \ldots, m$.

Proof We define ${ }^{2} \mathbf{B}: \mathbb{R}^{m} \times \mathbb{R}^{m} \rightarrow \mathbb{R}^{m, m}$ such that

$$
\mathbf{B}(\mathbf{z}, \mathbf{u}):=\int_{0}^{1} D \mathbf{F}(\mathbf{u}+t(\mathbf{z}-\mathbf{u})) d t, \quad \mathbf{z}, \mathbf{u} \in \mathbb{R}^{m} .
$$

We observe that $\mathbf{B}(\mathbf{z}, \mathbf{u})(\mathbf{z}-\mathbf{u})=\mathbf{F}(\mathbf{z})-\mathbf{F}(\mathbf{u})$. As a consequence, $\mathbf{z} \in \mathbf{S}(\mathbf{u})$ if and only if

$$
(\mathbf{B}(\mathbf{z}, \mathbf{u})-\sigma I)(\mathbf{z}-\mathbf{u})=\mathbf{0}
$$

for some $\sigma \in \mathbb{R}$. Since the system is strictly hyperbolic, $\mathbf{B}\left(\mathbf{u}_{l}, \mathbf{u}_{l}\right)=D \mathbf{F}\left(\mathbf{u}_{l}\right)$ has distinct eigenvalues, thus (due to Lemma 4) there exist a neighborhood $\mathcal{N}\left(\mathbf{u}_{l}, \mathbf{u}_{l}\right)$ and smooth functions $\lambda_{k}: \mathcal{N}\left(\mathbf{u}_{l}, \mathbf{u}_{l}\right) \rightarrow \mathbb{R}, \mathbf{r}_{k}: \mathcal{N}\left(\mathbf{u}_{l}, \mathbf{u}_{l}\right) \rightarrow \mathbb{R}^{m}$ and $\mathbf{l}_{k}: \mathcal{N}\left(\mathbf{u}_{l}, \mathbf{u}_{l}\right) \rightarrow$ $\mathbb{R}^{m}(k=1, \ldots, m)$ such that

$$
\left\{\begin{array}{l}
\mathbf{B}(\mathbf{z}, \mathbf{u}) \mathbf{r}_{k}(\mathbf{z}, \mathbf{u})=\lambda_{k}(\mathbf{z}, \mathbf{u}) \mathbf{r}_{k}(\mathbf{z}, \mathbf{u}) \\
\mathbf{B}^{T}(\mathbf{z}, \mathbf{u}) \mathbf{l}_{k}(\mathbf{z}, \mathbf{u})=\lambda_{k}(\mathbf{z}, \mathbf{u}) \mathbf{l}_{k}(\mathbf{z}, \mathbf{u})
\end{array}\right.
$$

for all $(\mathbf{z}, \mathbf{u}) \in \mathcal{N}\left(\mathbf{u}_{l}, \mathbf{u}_{l}\right)$. Furthermore, $\left\{\mathbf{r}_{k}(\mathbf{z}, \mathbf{u})\right\}_{k},\left\{\mathbf{l}_{k}(\mathbf{z}, \mathbf{v})\right\}_{k}$ are bases of $\mathbb{R}^{m}$ and $\mathbf{r}_{l}(\mathbf{z}, \mathbf{v}) \cdot \mathbf{l}_{k}(\mathbf{z}, \mathbf{v})=0$ if $l \neq k$.

\footnotetext{
2 This proof follows the same idea of the proof of [5, Theorem 2, Chapter 11.2.3].
} 
If we fix $k \in\{1, \ldots, m\}$, equation (13) holds if and only if $(\mathbf{z}-\mathbf{u}) \| \mathbf{r}_{k}(\mathbf{z}, \mathbf{u})$. As a consequence of the previous discussion, this condition can be reformulated as

$$
\begin{cases}\mathbf{l}_{l}(\mathbf{z}, \mathbf{u}) \cdot(\mathbf{z}-\mathbf{u})=0 & \text { if } l \neq k \\ \mathbf{l}_{k}(\mathbf{z}, \mathbf{u}) \cdot(\mathbf{z}-\mathbf{u}) \neq 0 & \text { if } l=k\end{cases}
$$

In view of the application of the Implicit Function Theorem, we define

$$
\boldsymbol{\Phi}_{k}: \mathbb{R}^{2 m} \rightarrow \mathbb{R}^{m-1} ; \quad \boldsymbol{\Phi}_{k}(\mathbf{z}, \mathbf{v})=\left[\begin{array}{c}
\vdots \\
\mathbf{l}_{k-1}(\mathbf{z}, \mathbf{u}) \cdot(\mathbf{z}-\mathbf{u}) \\
\mathbf{l}_{k+1}(\mathbf{z}, \mathbf{u}) \cdot(\mathbf{z}-\mathbf{u}) \\
\vdots
\end{array}\right] .
$$

The nonlinear system $\boldsymbol{\Phi}_{k}(\mathbf{z}, \mathbf{v})=\mathbf{0}$ consists of $m-1$ equations in $2 m$ variables. Let $\mathbf{z}^{\prime}=\left[z_{1}, \ldots, z_{m-1}\right]$ and $\tilde{\mathbf{l}}_{j}=\left[\left(\mathbf{l}_{j}\right)_{1}, \ldots,\left(\mathbf{l}_{j}\right)_{m-1}\right]^{T}$, we observe that

$\boldsymbol{\Phi}_{k}\left(\mathbf{u}_{l}, \mathbf{u}_{l}\right)=\mathbf{0}, \quad D_{\mathbf{z}^{\prime}} \boldsymbol{\Phi}_{k}(\mathbf{z}, \mathbf{u})=\left[\begin{array}{c}\vdots \\ \tilde{\mathbf{l}}_{k-1}^{T}(\mathbf{z}, \mathbf{u}) \\ \tilde{\mathbf{l}}_{k+1}^{T}(\mathbf{z}, \mathbf{u}) \\ \vdots\end{array}\right]+\left[\begin{array}{c}\vdots \\ \left(D_{\mathbf{z}^{\prime}} \mathbf{l}_{k-1}^{T}(\mathbf{z}, \mathbf{u})(\mathbf{z}-\mathbf{u})\right)^{T} \\ \left(D_{\mathbf{z}^{\prime}} \mathbf{l}_{k+1}^{T}(\mathbf{z}, \mathbf{u})(\mathbf{z}-\mathbf{u})\right)^{T} \\ \vdots\end{array}\right]$.

Since $\left\{\mathbf{l}_{k}(\mathbf{z}, \mathbf{u})\right\}_{k}$ is a basis of $\mathbb{R}^{m}, \operatorname{rank}\left(D_{\mathbf{z}^{\prime}} \boldsymbol{\Phi}_{k}\left(\mathbf{u}_{l}, \mathbf{u}_{l}\right)\right)=m-1$. Therefore, thanks to the Implicit Function Theorem, there exist a neighborhood $\mathcal{N}\left(\left(\mathbf{u}_{l}\right)_{m}, \mathbf{u}_{l}\right) \subset$ $\mathbb{R}^{m+1}$ and a $C^{1}$ function $\tilde{\boldsymbol{\Psi}}_{k}: \mathcal{N}\left(\left(\mathbf{u}_{l}\right)_{m}, \mathbf{u}_{l}\right) \rightarrow \mathbb{R}^{m}$ such that

$$
\left\{\begin{array}{l}
\mathbf{\Phi}_{k}\left(\tilde{\mathbf{\Psi}}_{k}(\mathbf{v}), \mathbf{v}\right)=0 \Leftrightarrow \tilde{\mathbf{\Psi}}_{k}(\mathbf{v}) \in \mathbf{S}_{k}(\mathbf{u}) \quad \forall \mathbf{v}=\left[\begin{array}{c}
s \\
\mathbf{u}
\end{array}\right] \in \mathcal{N}\left(\left(\mathbf{u}_{l}\right)_{m}, \mathbf{u}_{l}\right), \\
\tilde{\mathbf{\Psi}}_{k}\left(\left(\mathbf{u}_{l}\right)_{m}, \mathbf{u}_{l}\right)=\mathbf{u}_{l} .
\end{array}\right.
$$

The result follows by taking $\boldsymbol{\Psi}_{k}(t, \mathbf{u})=\tilde{\mathbf{\Psi}}_{k}\left(\left(\mathbf{u}_{l}\right)_{m}+t, \mathbf{u}\right)$.

We are now ready to prove our main result. We limit ourselves to the case $m=2$. The extension to the general case is not particularly complex.

Proof of Proposition 2 Given $\mathbf{u}_{l}$ and $\mathbf{u}_{r}$, we introduce the parameterizations $\mathbf{\Upsilon}_{k}: I \times \mathbb{R}^{2} \rightarrow \mathbb{R}^{2}$ for the curves $\mathbf{T}_{k}(\cdot), k=1,2$. Then, we introduce the function $\boldsymbol{\Phi}: \mathbb{R}^{6} \rightarrow \mathbb{R}^{2}$ such that $\boldsymbol{\Phi}(\boldsymbol{\epsilon}, \boldsymbol{\mu})=\boldsymbol{\Upsilon}_{2}\left(\epsilon_{2}, \boldsymbol{\Upsilon}_{1}\left(\epsilon_{1}, \mathbf{u}_{l}\right)\right)-\mathbf{u}_{r}$. Due to Lemma 5 , we have that $\boldsymbol{\Phi}(\boldsymbol{\epsilon}, \boldsymbol{\mu})$ is of class $C^{1}$. Furthermore,

$$
\begin{aligned}
D_{\boldsymbol{\epsilon}} \boldsymbol{\Phi}(\boldsymbol{\epsilon}, \boldsymbol{\mu}) & =\left[D_{\mathbf{u}} \mathbf{\Upsilon}_{2}\left(\epsilon_{2}, \mathbf{\Upsilon}_{1}\left(\epsilon_{1}, \mathbf{u}_{l}\right)\right) \partial_{\epsilon_{1}} \mathbf{\Upsilon}_{1}\left(\epsilon_{1}, \mathbf{u}_{l}\right), \partial_{\epsilon_{2}} \mathbf{\Upsilon}_{2}\left(\epsilon_{2}, \mathbf{\Upsilon}_{1}\left(\epsilon_{1}, \mathbf{u}_{l}\right)\right)\right] \\
& =\left[\mathbf{r}_{1}\left(\mathbf{u}_{l}\right), \mathbf{r}_{2}\left(\mathbf{u}_{l}\right)\right]+\mathcal{O}(\boldsymbol{\epsilon}),
\end{aligned}
$$

where we used the fact that $\left.D_{\mathbf{u}} \mathbf{\Upsilon}_{k}(\boldsymbol{\epsilon}, \mathbf{u})\right|_{\boldsymbol{\epsilon}=\mathbf{0}}=\mathbb{I}$ for $k=1$, 2. Since $\left\{\mathbf{r}_{k}\left(\mathbf{u}_{l}\right)\right\}_{k}$ is a basis of $\mathbb{R}^{2}$, we have that, for sufficiently small $\epsilon_{1}, \epsilon_{2}$,

$$
\operatorname{rank}\left(D_{\boldsymbol{\epsilon}} \boldsymbol{\Phi}(\boldsymbol{\epsilon}, \boldsymbol{\mu})\right)=2 .
$$

Applying the Implicit Function Theorem, we find that the solution $\epsilon^{\star}=\epsilon^{\star}(\boldsymbol{\mu})$ to $\boldsymbol{\Phi}\left(\boldsymbol{\epsilon}^{\star}, \boldsymbol{\mu}\right)=\mathbf{0}$ is differentiable in $\boldsymbol{\mu}$. Since the function $\boldsymbol{\Upsilon}_{1}$ is differentiable in both arguments (Lemma 5 ), then the state $\mathbf{u}_{1}$ defined by the formula $\mathbf{u}_{1}=\boldsymbol{\Upsilon}_{1}\left(\epsilon_{1}^{\star}, \mathbf{u}_{l}\right)$ is also differentiable in $\boldsymbol{\mu}$. The proof is complete. 


\section{Application to the $p$-System}

In this section, we apply our method to the $p$-system, which is a special case of (1a) representing a model for isentropic gas dynamics in Lagrangian coordinates. In Section 4.1, we introduce the problem and we derive the nonlinear system of algebraic equations (2) to be solved as well as a simplified system of the form (3). Then, in Section 4.2, we evaluate the performance of our method through a number of numerical experiments.

\subsection{The p-System}

We first introduce the mathematical formulation of the p-system. The p-system is a system of conservation laws of the form (1a) such that

$$
\mathbf{u}:=\left[\begin{array}{c}
w \\
v
\end{array}\right], \quad \mathbf{F}(w, v)=\left[\begin{array}{c}
-v \\
p(w)
\end{array}\right]
$$

where the function $p: \mathbb{R}_{+} \rightarrow \mathbb{R}$ satisfies
(i) $\lim _{w \rightarrow 0^{+}} p(w)=+\infty$,
(ii) $p^{\prime}(w)<0 \quad \forall w \in \mathbb{R}_{+}$,
(iii) $p^{\prime \prime}(w)>0 \quad \forall w \in \mathbb{R}_{+}$.

To simplify the notation, we define $a:=-p^{\prime}$.

Fixed $\mathbf{u}_{l} \in \mathbb{R}_{+}^{2}$, we now introduce the explicit formulas of the curves $\mathbf{T}_{k}\left(\mathbf{u}_{0}\right)$ in (6) for the p-system. First, we introduce the sets

$$
\begin{aligned}
& \mathbf{S}_{1}^{-}\left(\mathbf{u}_{l}\right):=\left\{(w, v) \in \mathbb{R}_{+}^{2}: w<w_{l}, v=\Phi_{1}\left(w, \mathbf{u}_{l}\right)=v_{l}-\sqrt{\left(p(w)-p\left(w_{l}\right)\right)\left(w_{l}-w\right)}\right\} \\
& \mathbf{S}_{2}^{-}\left(\mathbf{u}_{l}\right):=\left\{(w, v) \in \mathbb{R}_{+}^{2}: w>w_{l}, v=\Phi_{2}\left(w, \mathbf{u}_{l}\right)=v_{l}-\sqrt{\left(p(w)-p\left(w_{l}\right)\right)\left(w_{l}-w\right)}\right\}, \\
& \mathbf{R}_{1}^{+}\left(\mathbf{u}_{l}\right):=\left\{(w, v) \in \mathbb{R}_{+}^{2}: w>w_{l}, v=\Psi_{1}\left(w, \mathbf{u}_{l}\right)=v_{l}+\int_{w_{l}}^{w} \sqrt{a(s)} d s\right\} \\
& \mathbf{R}_{2}^{+}\left(\mathbf{u}_{l}\right):=\left\{(w, v) \in \mathbb{R}_{+}^{2}: w<w_{l}, v=\Psi_{2}\left(w, \mathbf{u}_{l}\right)=v_{l}-\int_{w_{l}}^{w} \sqrt{a(s)} d s\right\}
\end{aligned}
$$

and the corresponding curves $\mathbf{T}_{k}\left(\mathbf{u}_{l}\right)=\mathbf{R}_{k}^{+}\left(\mathbf{u}_{l}\right) \cup\left\{\mathbf{u}_{l}\right\} \cup \mathbf{S}_{k}^{-}\left(\mathbf{u}_{l}\right), k=1,2$. If we introduce the functions $\bar{\Upsilon}_{1}, \bar{\Upsilon}_{2} ; \mathbb{R} \times \mathbb{R}^{2} \rightarrow \mathbb{R}$ such that

$$
\bar{\Upsilon}_{1}\left(w, \mathbf{u}_{l}\right)=\left\{\begin{array}{ll}
\Phi_{1}\left(w, \mathbf{u}_{l}\right), & w<w_{l}, \\
\Psi_{1}\left(w, \mathbf{u}_{l}\right), & w \geq w_{l},
\end{array} \quad \bar{\Upsilon}_{2}\left(w, \mathbf{u}_{l}\right)= \begin{cases}\Psi_{2}\left(w, \mathbf{u}_{l}\right), & w<w_{l} \\
\Phi_{2}\left(w, \mathbf{u}_{l}\right), & w \geq w_{l}\end{cases}\right.
$$

we obtain that the maps $\epsilon \mapsto \Upsilon_{k}\left(\epsilon, \mathbf{u}_{l}\right)$,

$$
\mathbf{\Upsilon}_{1}\left(\epsilon, \mathbf{u}_{l}\right)=\left[\begin{array}{c}
w_{l}+\epsilon \\
\bar{\Upsilon}_{1}\left(w_{l}+\epsilon, \mathbf{u}_{l}\right)
\end{array}\right], \quad \mathbf{\Upsilon}_{2}\left(\epsilon, \mathbf{u}_{l}\right)=\left[\begin{array}{c}
w_{l}-\epsilon \\
\bar{\Upsilon}_{1}\left(w_{l}-\epsilon, \mathbf{u}_{l}\right)
\end{array}\right],
$$

are parametrizations of the curves $\mathbf{T}_{k}\left(\mathbf{u}_{l}\right)$ such that $\left\{\boldsymbol{\Upsilon}_{k}\left(\epsilon, \mathbf{u}_{l}\right): \epsilon>0\right\}=\mathbf{R}_{k}^{+}\left(\mathbf{u}_{l}\right)$ and $\left\{\boldsymbol{\Upsilon}_{k}\left(\epsilon, \mathbf{u}_{l}\right): \epsilon<0\right\}=\mathbf{S}_{k}^{-}\left(\mathbf{u}_{l}\right), k=1,2$. Finally, we introduce the partition 
$\left\{\mathcal{R}_{i}\left(\mathbf{u}_{l}\right)\right\}_{i=1}^{4}$ induced by the curves $\mathbf{T}_{k}\left(\mathbf{u}_{l}\right)$ :

$$
\begin{aligned}
& \mathcal{R}_{1}\left(\mathbf{u}_{l}\right)=\left\{(w, v) \in \mathbb{R}_{+}^{2}: w>w_{l}, \bar{\Upsilon}_{2}\left(w, \mathbf{u}_{l}\right)<v<\bar{\Upsilon}_{1}\left(w, \mathbf{u}_{l}\right)\right\} ; \\
& \mathcal{R}_{2}\left(\mathbf{u}_{l}\right)=\left\{(w, v) \in \mathbb{R}_{+}^{2}: v<\min \left\{\bar{\Upsilon}_{1}\left(w, \mathbf{u}_{l}\right), \bar{\Upsilon}_{2}\left(w, \mathbf{u}_{l}\right)\right\}\right\} ; \\
& \left.\mathcal{R}_{3}\left(\mathbf{u}_{l}\right)=\{w, v) \in \mathbb{R}_{+}^{2}: w<w_{l}, \bar{\Upsilon}_{1}\left(w, \mathbf{u}_{l}\right)<v<\bar{\Upsilon}_{2}\left(w, \mathbf{u}_{l}\right)\right\} ; \\
& \mathcal{R}_{4}\left(\mathbf{u}_{l}\right)=\left\{(w, v) \in \mathbb{R}_{+}^{2}: v>\max \left\{\bar{\Upsilon}_{1}\left(w, \mathbf{u}_{l}\right), \bar{\Upsilon}_{2}\left(w, \mathbf{u}_{l}\right)\right\}\right\} .
\end{aligned}
$$

Figure 1 shows the partition $\left\{\mathcal{R}_{i}\left(\mathbf{u}_{l}\right)\right\}_{i=1}^{4}$

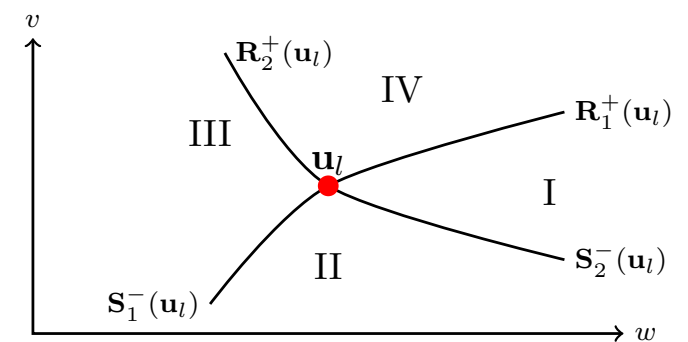

Fig. 1 State space partition (16a)-(16d) induced by the curves $\mathbf{T}_{k}\left(\mathbf{u}_{l}\right), k=1,2$

We can now present the main result of this section. We refer to [1, Section 5.5] for the proof.

Proposition 3 Should it exists, the solution to the Riemann problem (1a)-(1b) for the $p$-system $(14 \mathrm{a})-(14 \mathrm{~b})$ is uniquely determined by the solution $\widehat{\mathbf{u}}=\left(\epsilon_{1}^{\star}, \epsilon_{2}^{\star}, \mathbf{u}_{1}\right)$, $\mathbf{u}_{1}=\left(w_{1}, v_{1}\right)$, to the following system of equations

$$
\left\{\begin{array}{l}
\mathbf{u}_{1}-\Upsilon_{1}\left(\epsilon_{1}^{\star}, \mathbf{u}_{l}\right)=\mathbf{0} \\
\mathbf{u}_{r}-\mathbf{\Upsilon}_{2}\left(\epsilon_{2}^{\star}, \mathbf{u}_{1}\right)=\mathbf{0}
\end{array}\right.
$$

where $\boldsymbol{\Upsilon}_{1}, \mathbf{\Upsilon}_{2}$ have been introduced in (15). Furthermore, the solution to (17) is of the form

$$
\widehat{\mathbf{u}}=\left[\begin{array}{c}
w_{1}-w_{l} \\
w_{l}-w_{1} \\
w_{1} \\
v_{l}-h\left(w_{1}, w_{l}\right)
\end{array}\right],
$$

where $w_{1}=\widehat{u}^{\text {red }}$ is the unique solution to the nonlinear equation

$$
\mathcal{L}^{r e d}(w ; \boldsymbol{\mu})=v_{r}-v_{l}+h\left(w, w_{l}\right)+h\left(w, w_{r}\right)=0, \quad w \in \mathcal{I}_{i}(\boldsymbol{\mu}) .
$$

Here, the function $h: \mathbb{R}_{+}^{2} \rightarrow \mathbb{R}$ is defined as

$$
h\left(w, w_{K}\right):= \begin{cases}\sqrt{\left(p(w)-p\left(w_{K}\right)\right)\left(w_{K}-w\right)}, & w \leq w_{K}, \\ -\int_{w_{K}}^{w} \sqrt{a(s)} d s, & w>w_{K},\end{cases}
$$

where $K=l, r$, while the real interval $\mathcal{I}_{i}(\boldsymbol{\mu})$ is defined as

$$
\mathcal{I}_{i}(\boldsymbol{\mu}):= \begin{cases}\left(w_{l}, w_{r}\right), & i=1, \\ \left(0, \min \left\{w_{l}, w_{r}\right\}\right), & i=2, \\ \left(w_{r}, w_{l}\right), & i=3, \\ \left(\max \left\{w_{r}, w_{l}\right\}, \infty\right), & i=4\end{cases}
$$


4.2 Application of the Offline-Online Strategy to the p-System

In view of the numerical assessment, we first introduce a number of definitions. Given $h>0$, we introduce the parameter set

$$
\mathcal{D}_{h}:=\left\{\boldsymbol{\mu}=\left(\mathbf{u}_{l}, \mathbf{u}_{r}\right) \in \mathbb{R}^{4}: \mathbf{u}_{l}, \mathbf{u}_{r} \in[1,3]^{2},\left\|\mathbf{u}_{l}-\mathbf{u}_{r}\right\|_{\infty} \leq h\right\} .
$$

We also introduce the finite dimensional discretizations of $\mathcal{D}, \Xi_{N_{\text {on }}, h}^{\text {on }}=\left\{\boldsymbol{\mu}_{i}^{\text {on }}\right\}_{i=1}^{N_{\text {on }}}$ and $\Xi_{N_{\text {off }}, h}^{\text {off }}=\left\{\boldsymbol{\mu}_{i}^{\text {off }}\right\}_{i=1}^{N_{\text {off }}}$ : while the former is used to generate the surrogate model for the solution $\widehat{u}^{\text {red }}=w_{1}$ into $(18 \mathrm{~b})$ based on Algorithm 1, the latter is used to assess the performance of our approach. Both $\Xi_{N_{\text {off }}, h}^{\text {off }}$ and $\Xi_{N_{\text {on }}, h}^{\text {on }}$ are generated through a uniform random sampling over $\mathcal{D}$. In all our tests, we set $N_{\text {on }}=3000$, while we consider $N_{\text {train }}=0.5 N_{\text {off }}, N_{\text {val }}=N_{\text {test }}=0.25 N_{\text {off }}$. Furthermore, equation (18b) is solved using Newton's method with termination criterion based on the increment; the tolerance is set equal to $10^{-9}$. We also consider $p(w)=0.75 w^{-2.5}$. Finally, in order to compare our results with a benchmark, we introduce the $a$ priori surrogate $w_{1}^{\text {a priori }}$ defined as

$$
w_{1}^{\text {a priori }}(\boldsymbol{\mu}):= \begin{cases}\frac{1}{2}\left(w_{l}+w_{r}\right), & \mathbf{u}_{r} \in \mathcal{R}_{1}\left(\mathbf{u}_{l}\right) \cup \mathcal{R}_{3}\left(\mathbf{u}_{l}\right), \\ \frac{1}{2} \min \left\{w_{l}, w_{r}\right\}, & \mathbf{u}_{r} \in \mathcal{R}_{2}\left(\mathbf{u}_{l}\right), \\ \max \left\{w_{l}, w_{r}\right\}, & \mathbf{u}_{r} \in \mathcal{R}_{4}\left(\mathbf{u}_{l}\right) .\end{cases}
$$

In Fig. 2, we compare the accuracy of the surrogate model based on the offlineonline stage with the a priori surrogate (20). Figure 2(a) shows the behavior of the mean square error $M S E_{\text {off }}^{\text {surrogate }}$ computed offline based on (5) and of the mean square errors $M S E_{\mathrm{on}}^{\text {surrogate }}$ and $M S E_{\mathrm{on}}^{\mathrm{a} \text { priori }}$ computed online and defined by

$$
\left\{\begin{array}{l}
M S E_{\text {on }}^{\text {surrogate }}=\frac{1}{N_{\text {on }}} \sum_{i=1}^{N_{\text {on }}}\left(w_{1}\left(\boldsymbol{\mu}_{i}^{\text {on }}\right)-\widehat{u}^{\text {surrogate }}\left(\boldsymbol{\mu}_{i}^{\text {on }}\right)\right), \\
M S E_{\text {on }}^{\text {a priori }}=\frac{1}{N_{\text {on }}} \sum_{i=1}^{N_{\text {on }}}\left(w_{1}\left(\boldsymbol{\mu}_{i}^{\text {on }}\right)-w_{1}^{\text {a priori }}\left(\boldsymbol{\mu}_{i}^{\text {on }}\right)\right)
\end{array}\right.
$$

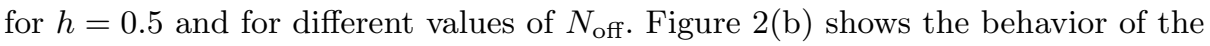
same quantities for $N_{\text {off }}=100$ and for different values of $h$. We observe that as $N_{\text {off }}$ increases, the accuracy of the surrogate model increases. We also observe that results weakly depend on the value of $h$.

In Fig. 3, we show the behavior of the average number of Newton's iterations for the two different choices of the initial guess. As in Fig. 2, in Fig. 3(a), we fix $h=0.5$ and we consider different values of $N_{\text {off }}$, while in Fig. 3(b), we fix $N_{\text {off }}=100$ and we consider different values of $h$. We observe that the improvement in the initial guess leads to a reduction of the required number of Newton's iterations for the same accuracy. This reduction motivates the additional offline expense required to train the surrogate model.

\section{Application to the Euler Equations for Ideal Gases}

In this section, we apply our method to the Euler equations for ideal gases. The discussion follows the same outline of Section 5. In Section 5.1, we present the problem statement, and we derive the reduced nonlinear algebraic system of equations (3) to be solved. Then, in Section 5.2, we present some numerical results. 


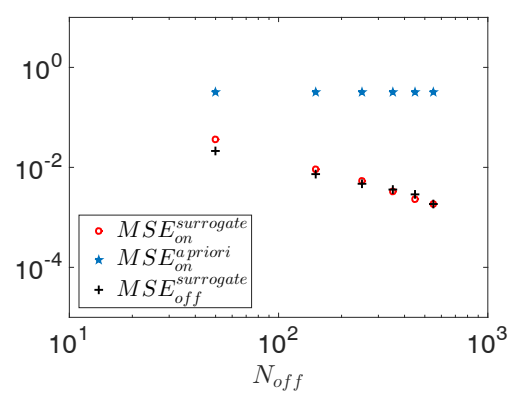

(a) Behavior of the mean square errors $M S E_{\text {off }}^{\text {surrogate }}, M S E_{\text {on }}^{\text {surrogate }}$ and $M S E_{\mathrm{on}}^{\mathrm{a} \text { priori }}$ with $N_{\text {off }}$ and for $h=0.5$

Fig. 2 Application to the p-system

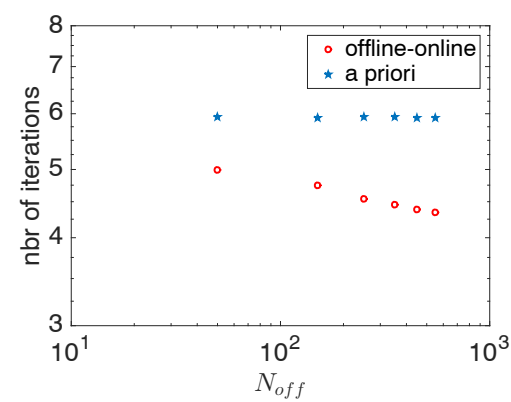

(a) Behavior of the average number of Newton's iterations with $N_{\text {off }}$ and for $h=0.5$

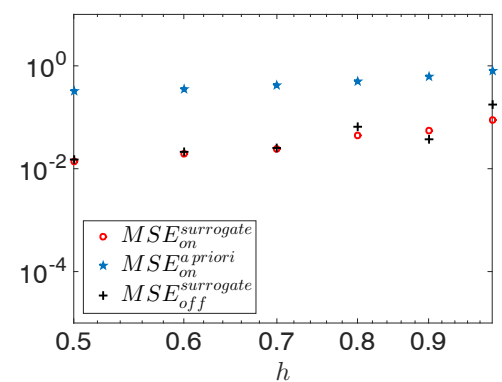

(b) Behavior of the mean square errors $M S E_{\text {off }}^{\text {surrogate }}, M S E_{\text {on }}^{\text {surrogate }}$ and $M S E_{\mathrm{on}}^{\mathrm{a}}{ }^{\text {priori }}$ with $h$ and for $N_{\mathrm{off}}=100$

Fig. 3 Application to the p-system

\subsection{Explicit formula for Euler Equations}

As in Section 4.1, we introduce the mathematical formulation of the problem of interest. The Euler equations for ideal gases are a system of conservation laws such that

$$
\mathbf{u}:=\left[\begin{array}{c}
\rho \\
\rho u \\
E
\end{array}\right], \quad \mathbf{F}(\mathbf{u}):=\left[\begin{array}{c}
\rho u \\
\rho u^{2}+p \\
u(E+p)
\end{array}\right] .
$$

Here, $\rho$ is the gas density, $u$ is the particle velocity, $p$ is the pressure, and $E$ is the total energy per unit volume defined as

$$
E=\frac{1}{2} \rho u^{2}+\frac{1}{\gamma-1} p
$$

with $\gamma>1$. We refer to $\mathbf{u}$ in $(22 \mathrm{a})$ as to the vector of conserved variables. For reasons that will become clear below, we also introduce the vector of primitive variables $\mathbf{w}=(\rho, u, p)$.

We now discuss the structure of the solution to the Riemann problem (1)-(22). We present the complete formula in Proposition 4. We refer to [16, Chapters 4.2 
and 4.4] for the proof. To simplify the statement, we present the solution in terms of the primitive variables.

Proposition 4 The solution to the Riemann problem (1)-(22) consists of four constant states $\mathbf{w}_{l}, \mathbf{w}_{1}, \mathbf{w}_{2}, \mathbf{w}_{r}$. The first two states, $\mathbf{w}_{l}, \mathbf{w}_{1}$, are separated by $a 1$ shock or by a 1-rarefaction wave, while $\mathbf{w}_{1}, \mathbf{w}_{2}$ are connected through a 2-contact discontinuity. Finally, $\mathbf{w}_{2}, \mathbf{w}_{r}$ are pieced together through a 3 -shock or a 3-rarefaction wave.

Furthermore, given $\mathbf{w}_{l}=\left[\rho_{l}, u_{l}, p_{l}\right]^{T}, \mathbf{w}_{r}=\left[\rho_{r}, u_{r}, p_{r}\right]^{T}$ and $\boldsymbol{\mu}=\left(\mathbf{w}_{l}, \mathbf{w}_{r}\right)$, we have that

$$
\mathbf{w}_{1}=\left[\begin{array}{c}
\rho_{\star}, l \\
u_{\star} \\
p_{\star}
\end{array}\right], \quad \mathbf{w}_{2}=\left[\begin{array}{c}
\rho_{\star, r} \\
u_{\star} \\
p_{\star}
\end{array}\right] .
$$

The pressure $p_{\star}=\widehat{u}^{\text {red }}$ is the unique solution to the nonlinear equation

$$
\mathcal{L}^{r e d}(p, \boldsymbol{\mu}):=f\left(p, \mathbf{w}_{l}\right)+f\left(p, \mathbf{w}_{r}\right)+u_{r}-u_{l}=0, \quad p \in \mathcal{I}(\boldsymbol{\mu}) .
$$

Here, the function $f: \mathbb{R} \times \mathbb{R}^{3} \rightarrow \mathbb{R}$ is defined by

$$
f\left(p, \mathbf{w}_{K}=\left[\rho_{K}, u_{K}, p_{K}\right]^{T}\right)= \begin{cases}\left(p-p_{K}\right) \sqrt{\frac{A\left(\rho_{K}\right)}{p+B\left(p_{K}\right)},} & p>p_{K}, \\ \frac{2 a\left(\rho_{K}, p_{K}\right)}{\gamma-1}\left[\left(\frac{p}{p_{K}}\right)^{\frac{\gamma-1}{2 \gamma}}-1\right], & p \leq p_{K},\end{cases}
$$

where $a(\rho, p)=\sqrt{\gamma \frac{p}{\rho}}$ is the speed of sound, $A(\rho)=\frac{2}{(\gamma+1) \rho}, B(p)=\frac{\gamma-1}{\gamma+1} p$ and $K=l, r$. The particle velocity $u_{\star}$ and the densities $\rho_{\star, l}$ and $\rho_{\star, r}$ are uniquely determined by $p_{\star}$ through the relations

$$
u_{\star}=\frac{1}{2}\left(u_{l}+u_{r}\right)+\frac{1}{2}\left(f\left(p_{\star}, \mathbf{w}_{r}\right)-f\left(p_{\star}, \mathbf{w}_{l}\right)\right),
$$

and

$$
\rho_{\star, K}= \begin{cases}\rho_{r}\left[\frac{\frac{\gamma-1}{\gamma+1}+\frac{p_{\star}}{p_{K}}}{\frac{\gamma-1}{\gamma+1} \frac{p_{\star}}{p_{K}}+1}\right], & p_{\star}>p_{K}, \\ \rho_{r}\left(\frac{p_{\star}}{p_{K}}\right)^{\frac{1}{\gamma}}, & p_{\star} \leq p_{K},\end{cases}
$$

with $K=l, r$. Finally, the interval $\mathcal{I}(\boldsymbol{\mu})$ is defined as

$$
\mathcal{I}(\boldsymbol{\mu}):= \begin{cases}\left(0, p_{\min }\right), & \mathcal{L}^{\text {red }}\left(p_{\min } ; \boldsymbol{\mu}\right)>0 \\ \left(p_{\min }, p_{\max }\right), & \mathcal{L}^{\text {red }}\left(p_{\min } \boldsymbol{\mu}\right)<0 \text { and } \mathcal{L}^{\text {red }}\left(p_{\max } ; \boldsymbol{\mu}\right)>0 \\ \left(p_{\max }, \infty\right), & \mathcal{L}^{\text {red }}\left(p_{\max } ; \boldsymbol{\mu}\right)>0\end{cases}
$$

where $p_{\min }=\min \left\{p_{l}, p_{r}\right\}$ and $p_{\max }=\max \left\{p_{l}, p_{r}\right\}$.

If $p_{\star}>p_{l}$, the states $\mathbf{w}_{l}$ and $\mathbf{w}_{1}$ are connected by a 1-shock, while if $p_{\star} \leq p_{l}$, the states $\mathbf{w}_{l}$ and $\mathbf{w}_{1}$ are connected by a 1-rarefaction wave. Similarly, if $p_{\star}>p_{r}$, the states $\mathbf{w}_{2}$ and $\mathbf{w}_{r}$ are connected by a 3-shock, while if $p_{\star} \leq p_{r}$, the states $\mathbf{w}_{2}$ and $\mathbf{w}_{r}$ are connected by a 3-rarefaction wave. 
5.2 Application of the Offline-Online Strategy to the Euler Equation

As in Section 4.2, given $h>0$, we introduce the parameter set

$$
\mathcal{D}_{h}:=\left\{\boldsymbol{\mu}=\left(\mathbf{u}_{l}, \mathbf{u}_{r}\right) \in \mathbb{R}^{6}: \mathbf{u}_{l}, \mathbf{u}_{r} \in[1,5]^{3},\left\|\mathbf{u}_{l}-\mathbf{u}_{r}\right\|_{\infty} \leq h\right\}
$$

and the finite dimensional discretizations $\Xi_{N_{\text {off }}, h}^{\text {off }}, \Xi_{N_{\text {on }}, h}^{\text {on }} \subset \mathcal{D}_{h}$. We set $N_{\text {on }}=$ $3000, N_{\text {train }}=0.5 N_{\text {off }}, N_{\text {val }}=N_{\text {test }}=0.25 N_{\text {off }}$ and we set the tolerance in Newton's method equal to $10^{-9}$. We also set $\gamma$ in $(22 \mathrm{~b})$ equal to 1.4 . Finally, we consider the a priori surrogate $p^{\text {a priori }}$ (see [16, (4.47) Chapter 4.3]) defined by

$p^{\text {a priori }}(\boldsymbol{\mu}):=\max \left\{T O L, p_{P V}(\boldsymbol{\mu})\right\}, \quad p_{P V}(\boldsymbol{\mu})=\frac{1}{2}\left(p_{l}+p_{r}\right)-\frac{1}{8}\left(u_{r}-u_{l}\right)\left(\rho_{r}+\rho_{l}\right)\left(a_{l}+a_{r}\right)$,

where $a_{K}=\sqrt{\gamma \frac{p_{K}}{\rho_{K}}}$ is the speed of sound, $K=l, r$. The tolerance $T O L$-here set equal to $10^{-3}$ - guarantees that the surrogate pressure is strictly positive. This surrogate is derived analytically from a linearized Riemann problem.

In Fig. 4, we compare the accuracy of the surrogate model based on the offlineonline stage with the a priori surrogate (25). Figure 4(a) shows the behavior of the mean square error $M S E_{\text {off }}^{\text {surrogate }}$ computed offline based on (5) and of the mean square errors $M S E_{\text {on }}^{\text {surrogate }}$ and $M S E_{\text {on }}^{\text {a priori }}$ computed online and defined as in

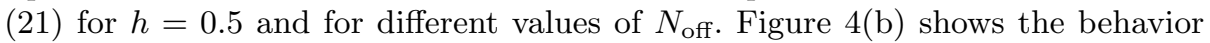
of the same quantities for $N_{\text {off }}=2000$ and for different values of $h$. As for the p-system, we observe that as $N_{\text {off }}$ increases, the accuracy of the surrogate model increases and that results weakly depend on the value of $h$.

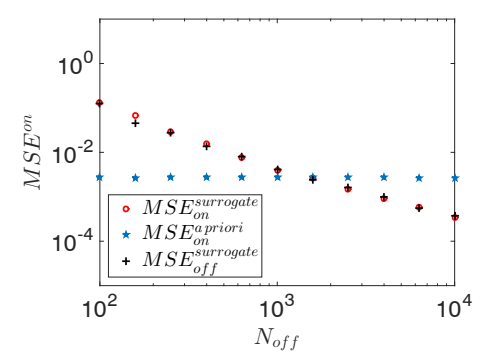

(a) Behavior of the mean square errors $M S E_{\text {off }}^{\text {surrogate }}, M S E_{\text {on }}^{\text {surrogate }}$ and $M S E_{\text {on }}^{\text {a priori }}$ with $N_{\text {off }}$ and for $h=0.5$

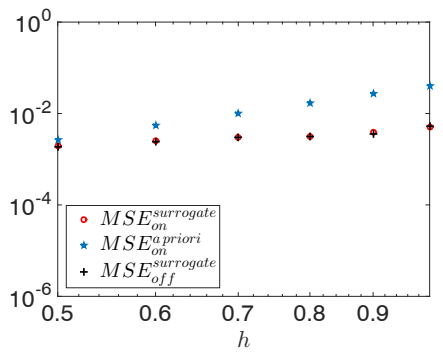

(b) Behavior of the mean square errors $M S E_{\text {off }}^{\text {surrogate }}, M S E_{\text {on }}^{\text {surrogate }}$ and $M S E_{\text {on }}^{\text {a priori }}$ with $h$ and for $N_{\text {off }}=2000$

Fig. 4 Application to the Euler equations

In Fig. 5, we show the behavior of the average number of Newton's iterations for the two different choices of the initial guess. As in Fig. 4, in Fig. 5(a), we fix $h=0.5$ and we consider different values of $N_{\text {off }}$, while in Fig. 3(b), we fix $N_{\text {off }}=2000$ and we consider different values of $h$. We observe that for this problem the improvement in the initial guess does not lead to a substantial reduction of the required number of Newton's iterations. 


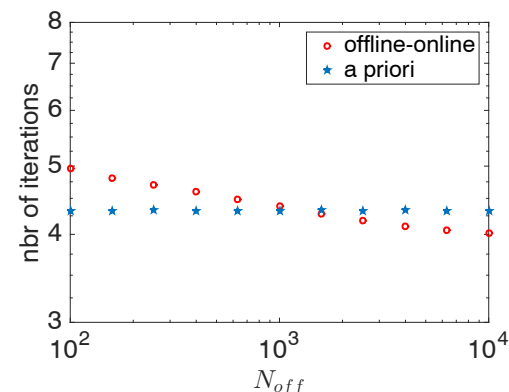

(a) Behavior of the average number of Newton's iterations with $N_{\text {off }}$ and for $h=0.5$

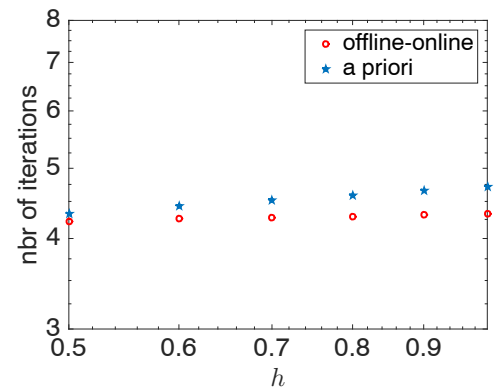

(b) Behavior of the average number of Newton's iterations with $h$ and for $N_{\text {off }}=2000$

Fig. 5 Application to the Euler equations

\section{Conclusions}

In this work, we propose an exact Riemann solver based on an offline-online computational decomposition. The approach is theoretically justified by the results presented in Section 3, and is applied to the p-system and to the Euler equations.

While for the p-system the improvement in the initial guess leads to a substantial reduction in the number of required Newton's iterations, for the Euler equations, the improvement is not substantial. However, we remark that the choice of the surrogate pressure (25) is absolutely not self-evident and is strictly related to the differential problem at hand. On the other hand, our "empirical" guess is computed automatically, and it is influenced by the equation only through the function $p^{\star}(\boldsymbol{\mu})$ and the parameter space $\mathcal{D}_{h}$.

As the next step, we wish to integrate this approach with traditional finite volume schemes for the approximation of nonlinear systems of conservation laws with arbitrary initial and boundary conditions.

Acknowledgements The authors thank the unknown reviewers for their insightful comments that help improve the manuscript.

\section{References}

1. Bressan, A.: Hyperbolic Systems of Conservation Laws-The One-Dimensional Cauchy Problem. Oxford Univ. Press, Oxford (2000)

2. Carlberg, K., Farhat, C., Cortial, J., Amsallem, D.: The GNAT method for nonlinear model reduction: effective implementation and application to computational fluid dynamics and turbulent flows. J. Comput. Phys. 242, 623-647 (2013)

3. Carlberg, K., Ray, J., van Bloemen Waanders, B.: Decreasing the temporal complexity for nonlinear, implicit reduced-order models by forecasting. Comput. Methods Appl. Mech. Eng. 289, 79-103, (2015)

4. Chorin, A.J.: Random choice solution of hyperbolic systems. J. Comput. Phys. 22, 517-533 (1976)

5. Evans, L.C.: Partial Differential Equations. Graduate Studies in Mathematics, Vol. 19, 2nd edn. American Mathematical Society, Providence (2010)

6. Godunov, S.K.: A finite difference method for the computation of discontinuous solutions of the equations of fluid dynamics. Mat. Sb 47, 357-393 (1959) 
7. Gottlieb, J.J., Groth, C.P.T.: Assessment of Riemann solvers for unsteady one-dimensional inviscid flows of perfect gases. J. Comput. Phys. 78, 437-458 (1988)

8. Hesthaven, J.S., Stamm, B., Zhang, S.: Efficient greedy algorithms for high-dimensional parameter spaces with applications to empirical interpolation and reduced basis methods. ESAIM Math. Model. Numer. Anal. 48, 259-283 (2014)

9. Kunisch, K., Volkwein, S.: Galerkin proper orthogonal decomposition methods for a general equation in fluid dynamics. SIAM J. Numer. Anal. 40, 492-515 (2002)

10. Lax, P.D.: Hyperbolic Systems of Conservation Laws and the Mathematical Theory of Shock Waves. SIAM (1973)

11. Murphy, K.P.: Machine Learning: A Probabilistic Perspective. MIT Press (2012)

12. Nocedal, J., Wright, S.: Numerical Optimization. Springer (2006)

13. Quarteroni, A., Manzoni, A., Negri, F.: Reduced Basis Methods for Partial Differential Equations: An Introduction. Springer (2015)

14. Roe, P.L.: Approximate Riemann solvers, parameter vectors, and difference schemes. J. Comput. Phys. 43, 357-372 (1981)

15. Rubinstein, R.Y., Kroese, D.P.: Simulation and the Monte Carlo Method. John Wiley \& Sons (2011)

16. Toro, E.F.: Riemann Solvers and Numerical Methods for Fluid Dynamics: A Practical Introduction. Springer, Berlin (1997)

17. Van Leer, B.: Towards the ultimate conservative difference scheme. IV. A new approach to numerical convection. J. Comput. Phys. 23, 276-299 (1977) 